\title{
Spatial Convergence of Three Dimensional Turbulent Flows
}

\author{
Michael A. Park* and W. Kyle Anderson ${ }^{\dagger}$ \\ NASA Langley Research Center, Hampton, VA 23681, USA
}

\begin{abstract}
Finite-volume and finite-element schemes, both implemented within the FUN3D flow solver, are evaluated for several test cases described on the Turbulence-Modeling Resource (TMR) web site. The cases include subsonic flow over a hemisphere cylinder, subsonic flow over a swept bump configuration, and supersonic flow in a square duct. The finitevolume and finite-element schemes are both used to obtain solutions for the first two cases, whereas only the finite-volume scheme is used for the supersonic duct. For the hemisphere cylinder, finite-element solutions obtained on tetrahedral meshes are compared with finitevolume solutions on mixed-element meshes. For the swept bump, finite-volume solutions have been obtained for both hexahedral and tetrahedral meshes and are compared with finite-element solutions obtained on tetrahedral meshes. For the hemisphere cylinder and the swept bump, solutions are obtained on a series of meshes with varying grid density and comparisons are made between drag coefficients, pressure distributions, velocity profiles, and profiles of the turbulence working variable. The square duct shows small variation due to element type or the spatial accuracy of turbulence model convection. It is demonstrated that the finite-element scheme on tetrahedral meshes yields similar accuracy as the finitevolume scheme on mixed-element and hexahedral grids, and demonstrates less sensitivity to the mesh topology (biased tetrahedral grids) than the finite-volume scheme.
\end{abstract}

\section{Introduction}

The use of Reynolds-averaged Navier-Stokes (RANS) with a turbulence model has become a critical tool for the design of aerospace vehicles. However, the issues that affect the grid convergence of three dimensional (3D) configurations are not completely understood, as documented in the AIAA Drag Prediction Workshop series. ${ }^{1-3}$ This effort extends a previous grid convergence study of turbulent two dimensional (2D) flows. ${ }^{4}$ In particular, the 3D Bump-in-channel, 3D Supersonic Square Duct, and 3D Hemisphere Cylinder cases from the Turbulence Modeling Resource (TMR) $)^{5-7}$ will be examined.

\section{Spatial Discretization and Time Advancement}

For the current work, the spatial accuracy of finite-volume and finite-element schemes are examined, both of which are implemented in the FUN3D unstructured-grid RANS flow solver. For each scheme, a brief summary of each discretization is given below, along with a short description of the time advancement algorithm used for obtaining steady-state solutions.

\section{II.A. Finite-Volume Discretization}

The finite-volume discretization is described by Anderson and Bonhaus ${ }^{8}$ and Nielsen. ${ }^{9}$ The flow variables are stored at the vertices of the mesh. FUN3D solves the equations on mixed-element grids, including tetrahedra, pyramids, prisms, and hexahedra. At interfaces delimiting neighboring control volumes, the inviscid fluxes are computed using an approximate Riemann solver based on the values on either side of the interface. Roe's flux-difference-splitting scheme ${ }^{10}$ is used in the current study. For second-order accuracy, interface values are obtained by a U-MUSCL scheme ${ }^{11,12}$ with gradients reconstructed at the mesh vertexes using an unweighted

\footnotetext{
${ }^{*}$ Research Scientist, Computational AeroSciences Branch, AIAA Senior Member.

${ }^{\dagger}$ Research Scientist, Computational AeroSciences Branch, AIAA Associate Fellow.
} 
least-squares technique. ${ }^{8}$ The U-MUSCL scheme coefficient $\chi$ is set to 0.0 for purely tetrahedral grids and 0.5 for grids that contain other element types.

For tetrahedral meshes, the full viscous fluxes are discretized using a finite-volume formulation in which the required velocity gradients on the dual faces are computed using the Green-Gauss theorem applied to the elements that surround the dual faces. On tetrahedral meshes this is equivalent to a Galerkin type approximation. For non-tetrahedral meshes, the same Green-Gauss approach can lead to odd-even decoupling. A pure edge-based approach can be used to circumvent the odd-even decoupling issue but yields only approximate viscous terms. Therefore, for non-tetrahedral meshes, the edge-based gradients are combined with Green-Gauss gradients; this improves the h-ellipticity of the operator and allows the complete viscous stresses to be evaluated. ${ }^{8,13}$

The Spalart-Allmaras ${ }^{14}(\mathrm{SA})$ model is solved loosely coupled to the mean-flow equations. The negative SA model ${ }^{15}$ (SA-neg) is available as well as the original model. The SA-neg model is identical to the original SA model for positive values of the turbulence working variable. The SA-neg model exhibits better iterative convergence properties, especially for initial transients. With the finite-volume discretization, both first-order or second-order approximations for the convection term in the turbulence-model equation can be utilized. The quadratic constitutive relation (SA-neg-QCR2000) modification of Spalart ${ }^{16}$ is also applied to the square duct example.

The solution at each time-step is updated with a backwards Euler time-differencing scheme. On the innermost level it uses a preconditioner based on a defect-correction method and iterates on a simplified Jacobian with first-order convection terms, exact diffusion terms, and a pseudo-time term. At each time step, the linear system of equations is approximately solved with a multi-color point-implicit procedure. Local time-step scaling is employed to accelerate convergence to steady-state. This implicit relaxation scheme can also be used as a preconditioner to Generalized Conjugate Residual (GCR) ${ }^{17}$ for stabilization and convergence acceleration.

A nonlinear controller assesses the correction computed by the linear solver. The controller is responsible for the CFL adaptation strategy and for deciding when to update the Jacobian. As a result of this assessment, the suggested correction can be applied fully, partially, or completely discarded; the current Jacobian may be updated or reused in the next iteration; and the current CFL number may increase, decrease, or stay the same. Details of the adaptive time advancement scheme are provided by Pandya et al. ${ }^{18}$

\section{II.B. Finite-Element Discretization}

A finite-element scheme is currently under development within FUN3D. The discretization is based on a stabilized finite-element approach that includes the Streamlined Upwind Petrov-Galerkin (SUPG) scheme, ${ }^{19,20}$ Galerkin least squares, ${ }^{21}$ and variational multiscale methods. ${ }^{22}$ In the results shown here, only the SUPG scheme is considered.

In the current implementation, the negative SA turbulence model ${ }^{15}$ is tightly coupled with the flow equations, yielding a non-linear algebraic system of equations with six variables at each mesh point. Although the current implementation includes the capability for computing on tetrahedra, hexahedra, pyramids, and prisms, only results for tetrahedral meshes are included.

To advance the solution towards a steady state, the density, velocities, temperature, and the turbulence working variable are updated in a tightly-coupled formulation using a Newton-type solver similar to Burgess and Glasby. ${ }^{23}$ Here, an initial update to the flow variables is computed using Newton's method, with a locally varying time-step parameter used to help provide global convergence. This full Newton update is limited so that the density and temperature are not allowed to change more than fifteen percent. This limit is then used to provide an upper bound for a line search to determine an optimal value. The root mean square (RMS) of the residual is determined at four under-relaxed updates between zero and the maximum update limit determined from realizability conditions described above. Using the four RMS values of the residual, the optimal relaxation factor is determined by locating the minimum of a cubic polynomial curve fit through the RMS samples.

At each iteration, the linear system is solved using the generalized minimal residual method (GMRES) ${ }^{24}$ algorithm with a preconditioner based on an incomplete lower upper (LU) decomposition with two levels of fill ${ }^{25}$ and a Krylov subspace dimension of 200. It should be noted that these parameters are based on previous experience for $2 \mathrm{D}$ problems ${ }^{26}$ but no extensive studies have been conducted to determine optimal parameters for 3D problems. 
The solution variables used for the finite-element discretization differ from those normally stored in the perfect gas compressible FUN3D finite-volume discretization, which uses nondimensional conserved variables. The choice in solving for temperature directly in the finite-element discretization has been made to facilitate future computations of real-gas flows where the equation of state is invariably given directly in terms of density and temperature. At the completion of the solution process, the finite-element solution variables are converted to conserved variables and passed back into the primary solver, which subsequently computes the forces using previously developed finite-volume routines. It should be noted that this process may adversely impact the accuracy of the force computations, which should be computed directly from the flux routines within the finite-element portion of the code.

\section{Test Cases}

Three test cases are examined to evaluate the relative accuracy of the finite-volume and finite-element discretizations. A brief description and relevant results are provided below for each case. Further details for the test cases are provided on the TMR web site. For the cases presented, the residual RMS are converged below $10^{-12}$ for all equations.

\section{III.A. 3D Hemisphere Cylinder}

The first test case is a hemisphere cylinder that was experimentally tested by Hsieh. ${ }^{27}$ A general overview of the model is depicted in Fig. 1, which shows a surface mesh on the left, and typical pressure contours on the right. The flow conditions correspond to a free-stream Mach number of 0.6, an angle-of-attack of zero degrees, and a Reynolds number of 4.2 million per foot (350,000 per inch). The computational meshes extend ten units in the axial direction, which corresponds to the length of the wind-tunnel model in inches. Therefore, with the current meshes, the simulations are run at a free stream Reynolds number of 350,000 to provide conditions consistent with the experiment.

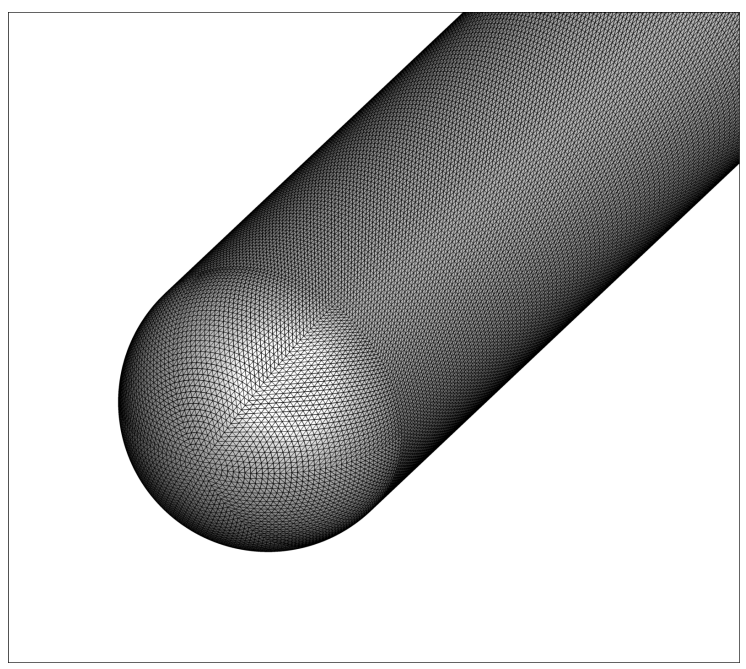

(a) Tetrahedral mesh for hemisphere cylinder.

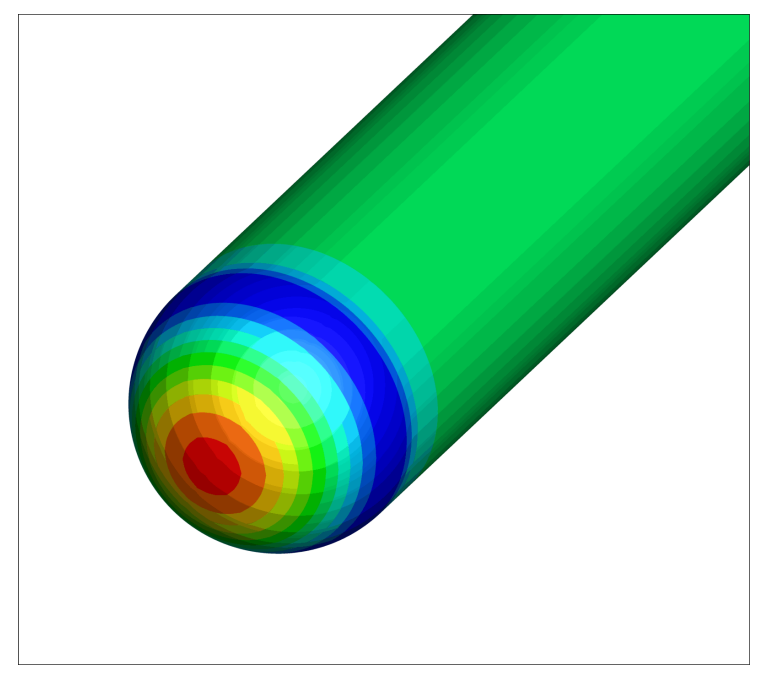

(b) Pressure coefficients.

Figure 1. Surface mesh and contours of pressure coefficient for hemisphere cylinder.

For this configuration, finite-volume computations have been conducted by Diskin et al. ${ }^{28}$ on very fine meshes with the intent of providing reference solutions for other researchers. The unstructured meshes used for these simulations range in density from approximately 2 million nodes to just under 126 million nodes. The finest mesh has been generated using an algebraic technique embodied in a Fortran code available on the TMR web site. Each coarser mesh is generated using an additional program that removes alternate mesh lines from an otherwise structured topology, thereby generating a nested family of grids. A summary of the grid statistics, including the number of nodes, the number of hexahedra and prisms, the estimated wall coordinate for the first grid point off the wall, and the number of nodes axially located on the surface of the geometry is given in Table 1 .

One purpose of the current work is to examine the effects of using tetrahedral elements on the accuracy 
Table 1. Family of mixed-element grids for finite-volume solutions.

\begin{tabular}{lrrrccc} 
Grid Level & Nodes & Hexahedra & Prisms & $h \approx(1 / N)^{1 / 3}$ & $\mathrm{y}+$ & Axial nodes \\
\hline level 2 & $2,010,998$ & $1,776,000$ & 355,200 & 0.0079 & 2.0 & 200 \\
level 1 & $15,858,075$ & $14,208,000$ & $2,841,600$ & 0.0049 & 1.0 & 400 \\
level 0 & $125,946,869$ & $113,664,000$ & $22,732,800$ & 0.0020 & 0.5 & 800
\end{tabular}

of the solutions obtained using both the finite-volume and finite-element discretizations. To this end, a series of tetrahedral meshes has been generated, also using the code from the TMR web site. In this case, however, the sequence of coarser grids is not generated by removing alternate mesh lines from the finest mesh. Instead, the finest mesh is generated with 400 nodes distributed in the axial location along the surface of the cylinder with a wall coordinate of 1.0. The coarser meshes are then generated by subsequent halving of the number of nodes along the axial direction, and doubling the spacing normal to the wall. A final summary of the tetrahedral meshes is provide in Table 2. As seen in the table, the finest mesh consists of 18.6 million nodes and 110 million tetrahedra, whereas the coarsest mesh only has 371,308 nodes and just over 2 million tetrahedra.

Table 2. Family of tetrahedral grids for finite-volume and finite-element solutions.

\begin{tabular}{lrrccc} 
Grid Level & Nodes & Tetrahedra & $h \approx(1 / N)^{1 / 3}$ & $\mathrm{y}+$ & Axial nodes \\
\hline level C & 371,308 & $2,138,400$ & 0.0139 & 4.0 & 200 \\
level B & $2,698,971$ & $15,840,000$ & 0.0072 & 2.0 & 400 \\
level A & $18,606,808$ & $110,246,400$ & 0.0038 & 1.0 & 800
\end{tabular}

In the comparisons that follow, the results obtained on the finest mesh from Diskin et al. ${ }^{28}$ are used as datum solutions for comparison. The pressure distribution along the upper surface of the cylinder is examined, as are profiles of both the $u$-component of velocity and the turbulence model working variable. The variation of the total drag coefficient is also examined as a function of mesh density. These results are further broken down into appropriate contributions from pressure forces and skin friction forces to provide more detailed analysis.

Figure 2 shows the pressure distributions obtained with the finite-element and finite-volume schemes compared to the reference solution, as well as with experiment. Figure 2(a) demonstrates that very little differences are evident from the solutions obtained on the finest and coarsest meshes when computed using the finite-element discretization on a tetrahedral mesh. Furthermore, the finite-element solutions agree very well with the finite-volume reference solution obtained on the mixed-element mesh with almost 126 million

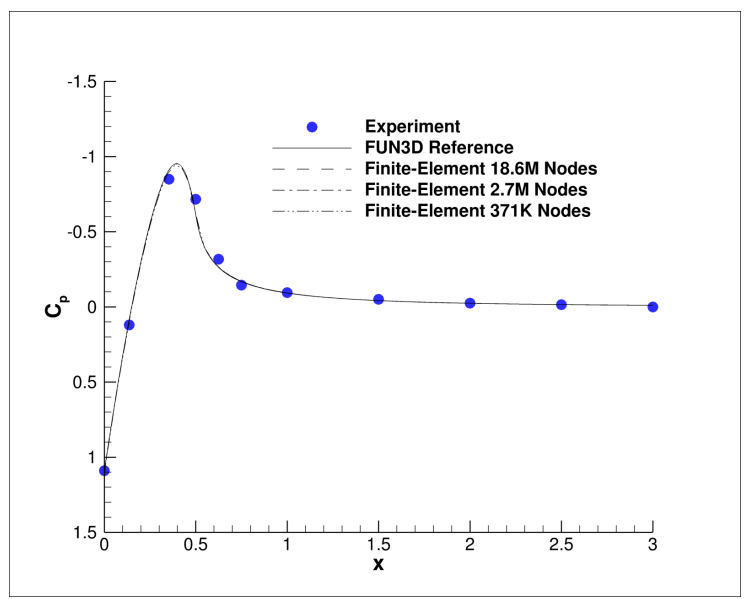

(a) Pressure coefficients from finite-element discretization.

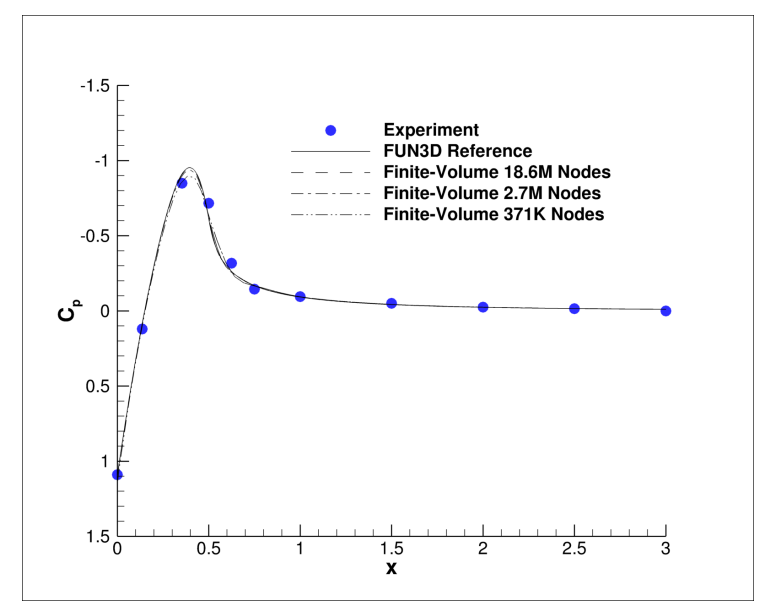

(b) Pressure coefficients from finite-volume discretization.

Figure 2. Pressure Distribution for Hemisphere Cylinder. 
nodes. In Fig. 2(b), which shows results obtained using the finite-volume scheme, the pressure distributions obtained on the different meshes are also very similar, although there is slightly more difference between the solution obtained on the coarsest mesh and the reference solution than was evident in the finite-element solution.

In Fig. 3, the skin-friction coefficients obtained with the finite-element and finite-volume schemes on the tetrahedral meshes are compared with the finite-volume reference solution. In Fig. 3(a), the agreement between finite-element and reference solutions is very good for the fine grid and the medium grid, but a discrepancy is apparent on the coarsest mesh of 371 thousand nodes. Figure 3(b) shows results obtained using the finite-volume discretization. As with the finite-element results, the comparison between the finegrid solution and the reference solution is very good, although slightly more difference is observed in the solutions on the medium and coarse meshes.

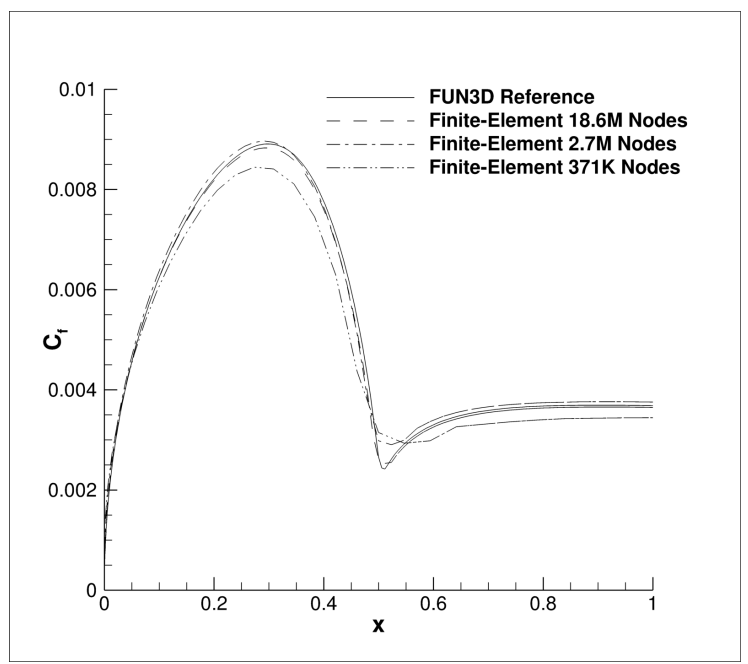

(a) Skin-friction coefficients from finite-element discretization.

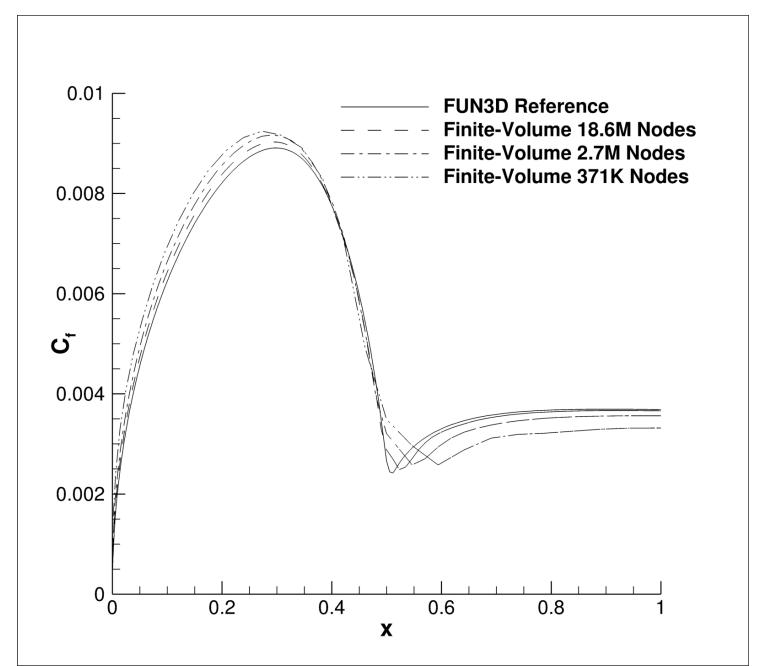

(b) Skin-friction coefficients from finite-volume discretization.

Figure 3. Skin-Friction Distribution for Hemisphere Cylinder.

Profiles of the $u$-component of velocity at the $x / L=0.05(x=0.5)$ intersection of the hemisphere and cylinder are shown in Fig. 4, which presents comparisons of the finite-element and finite-volume results obtained on each mesh with the reference solution. In Fig. 4(a), the solutions obtained with the finiteelement and finite-volume discretizations agree well with the reference solution. Note that the discrepancies that appear between $y=0.0010$ and $y=0.0015$ are simply because the solution within the elements is assumed to be linear. On the medium and coarse mesh, the differences between the current solutions and the reference solution increase as the mesh density decreases. However, it should be noted that the finiteelement solution is closer to the reference solution, indicating that the solution is slightly more accurate.

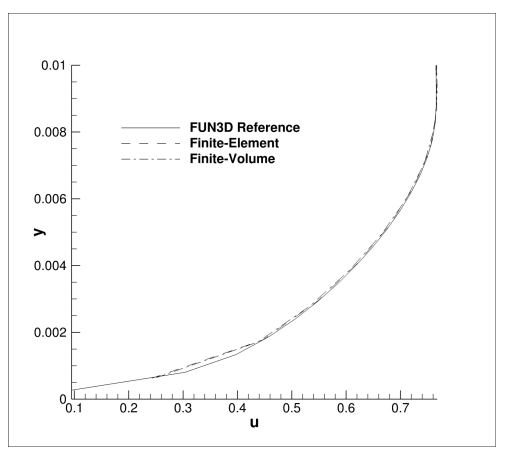

(a) Fine mesh.

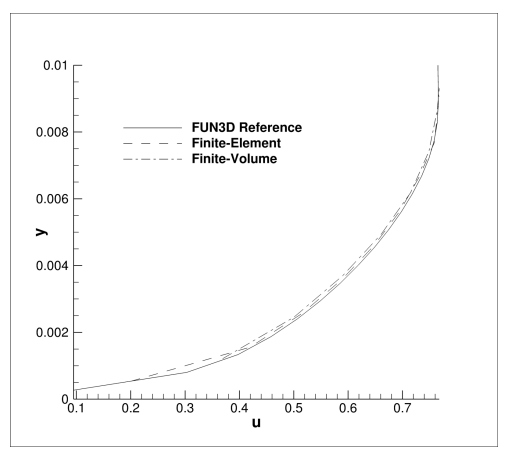

(b) Medium mesh.

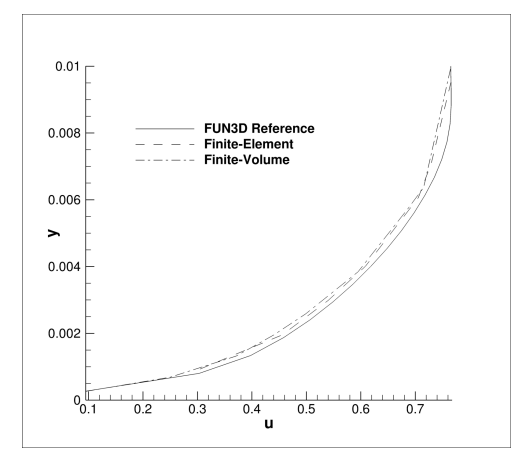

(c) Coarse mesh.

Figure 4. Profile of $u$-velocity.

Profiles of the turbulence working variable at the $x / L=0.05(x=0.5)$ intersection of the hemisphere 
and cylinder, depicted in Fig. 5, show similar trends as observed with the velocity profiles. Specifically, on the finest tetrahedral mesh, the finite-element and finite-volume solutions both agree well with the reference solution obtained using the finite-volume scheme on a mixed-element mesh with approximately 126 million nodes. As with the velocity profiles, differences between the current solutions and the reference solution increase as the mesh is coarsened, and the finite-element solution appears to be somewhat more accurate than the finite-volume solution obtained on the same mesh.

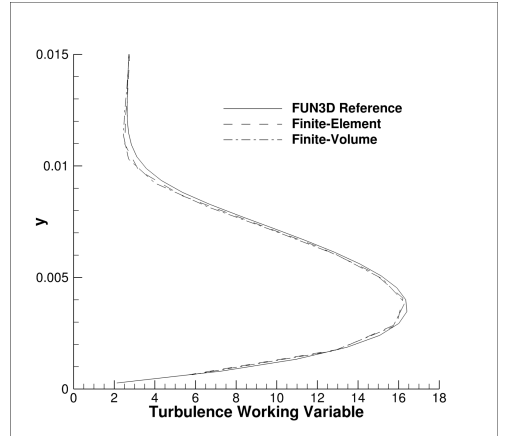

(a) Fine mesh.

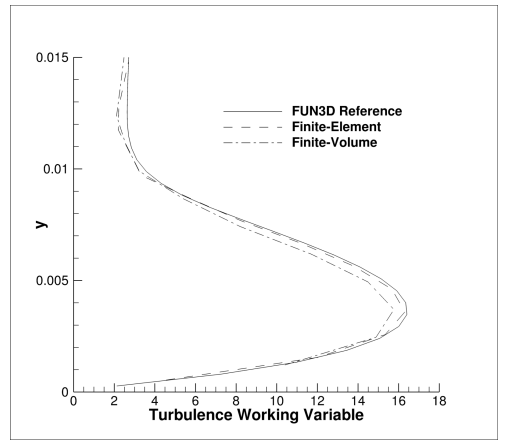

(b) Medium mesh.

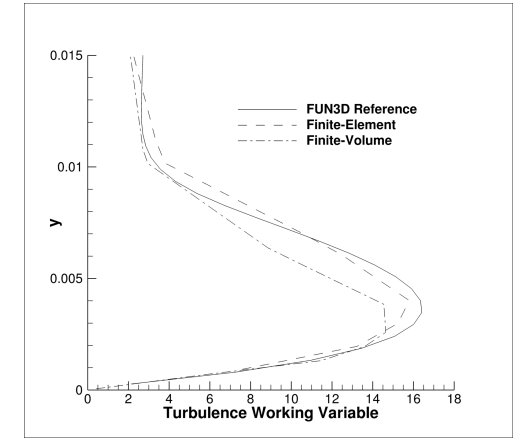

(c) Coarse mesh.

Figure 5. Profile of turbulence working variable.

Finally, the convergence of the drag coefficient obtained on the above sequence of meshes is shown in Fig. 6. A reference area of ten has been used in the computation of the drag coefficient to be consistent with that used in establishing the reference solutions. ${ }^{28}$ In Fig. 6, the total drag, pressure drag, and skinfriction drag are shown for the finite-element and finite-volume results compared with that of the reference solutions. In Fig. 6, the reference solutions are depicted with solid circles for easier identification. All the solutions, including the reference solution, are still exhibiting noticeable changes as the mesh is continually refined. However, the changes in the drag between meshes decreases with each mesh refinement, as expected. In examining the total drag and the pressure drag, the finite-element scheme shows less sensitivity between meshes, and is generally closer to the asymptotic value for a given number of degrees of freedom. In contrast, the skin-friction drag from the finite-element solution exhibits a inexplicable non-monotone behavior where the viscous drag is lower on the medium mesh than either the fine or coarser ones.

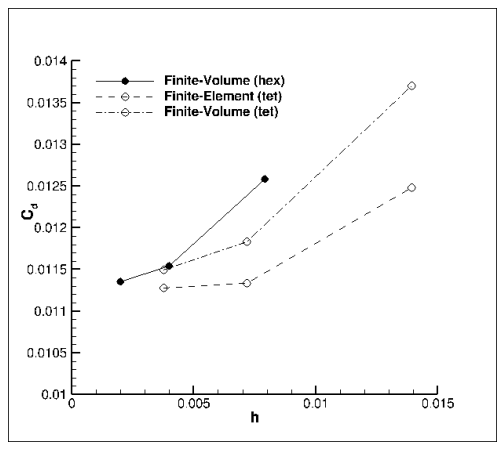

(a) Total drag.

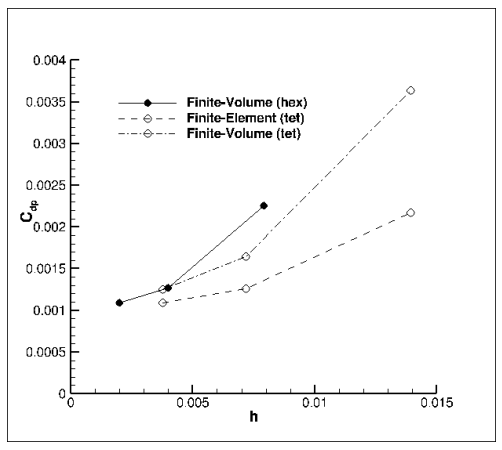

(b) Pressure drag.

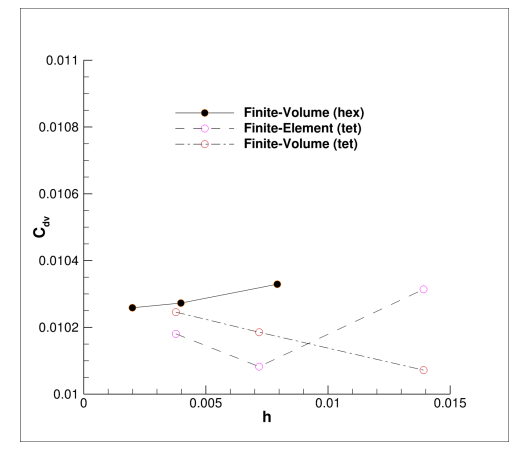

(c) Skin-friction drag.

Figure 6. Drag convergence.

\section{III.B. 3D Bump}

The next test case is a swept 3D bump which is also described in further detail on the TMR web site. For this case, finite-element and finite-volume solutions are obtained on pure tetrahedral meshes and compared with finite-volume solutions obtained on hexahedra meshes. Solutions have been obtained on hexahedral and tetrahedral meshes ranging from just over 18 thousand nodes, up to over 966 thousand nodes. Comparisons are made with simulations by Diskin et al. ${ }^{28}$ that have been computed on hexahedral meshes with almost 59 million nodes. The 3D bump depicted in Fig. 7 shows the surface mesh for representative tetrahedral and hexahedral meshes, respectively. The flow conditions correspond to a free-stream Mach number of 0.2 and 
a Reynolds number of 3 million based on unit grid length. In the results that follow, comparisons are again made with the results described in Diskin et al. ${ }^{28}$ for drag coefficients, pressure distributions, and profiles of $u$-velocity and the turbulence working variable.

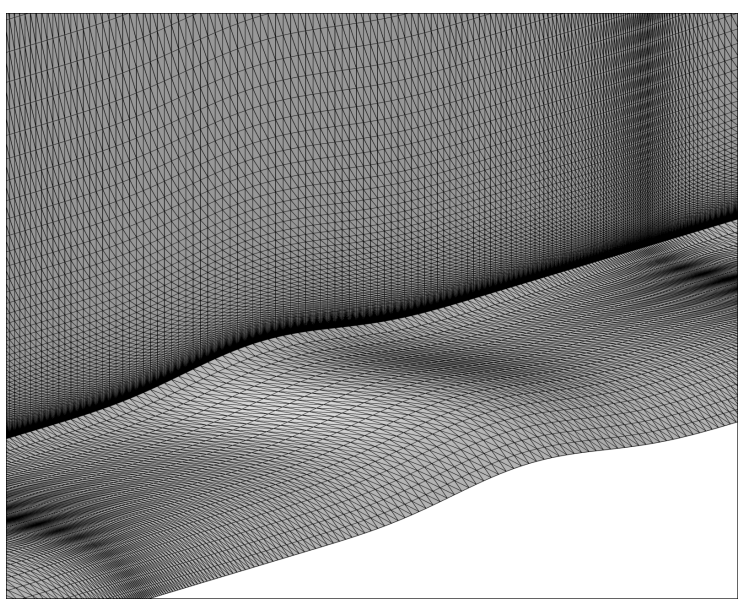

(a) Tetrahedral mesh for swept bump.

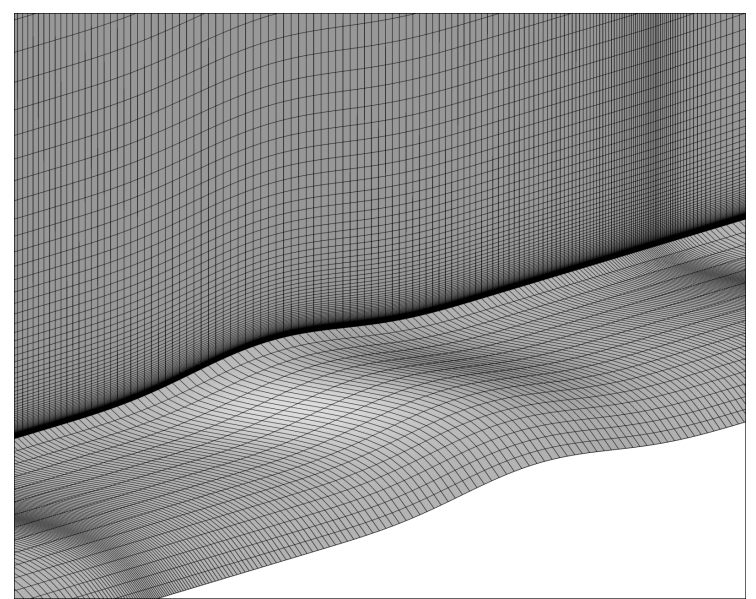

(b) Hexahedral mesh for swept bump.

Figure 7. Geometry and surface meshes for tetrahedral and hexahedral grids.

A summary of the mesh sizes used for this study is given in Table 3. Note that for the finest mesh, finitevolume results on hexahedra from Diskin et al. ${ }^{28}$ are used as reference solutions. Because the tetrahedra were not used beyond mesh sizes of 966 thousand nodes, the number of tetrahedra for the finest two meshes is designated with "NA". Pressure distributions, velocity profiles, and profiles of the turbulence working variable computed using the finite-element and finite-volume results on tetrahedral meshes have been obtained on grid levels $3-5$, and are compared to corresponding finite-volume results on hexahedral meshes of the same density.

Table 3. Family of tetrahedral grids for finite-volume and finite-element solutions.

\begin{tabular}{lrrrr} 
Grid Level & Nodes & Hexahedra & Tetrahedra & $h \approx(1 / N)^{1 / 3}$ \\
\hline level 5 & 18,245 & 14,080 & 84,480 & 0.0380 \\
level 4 & 129,033 & 112,640 & 675,840 & 0.0198 \\
level 3 & 966,161 & 901,120 & $5,406,720$ & 0.0101 \\
level 2 & $7,468,065$ & $15,840,000$ & $\mathrm{NA}$ & 0.0051 \\
level 1 & $58,705,985$ & $110,246,400$ & $\mathrm{NA}$ & 0.0026
\end{tabular}

Computed contours of pressure coefficients over the surface of the bump are shown in Fig. 8, Fig. 9, and Fig. 10. The contours shown in Fig. 8 have been obtained with the finite-volume scheme on hexahedral meshes. The contours shown in Fig. 9 depict solutions obtained on tetrahedral meshes using the finiteelement scheme and the contours shown in Fig. 10 depict solutions obtained on tetrahedral meshes using the finite-volume scheme. As seen in Fig. 8 and Fig. 9, the finite-volume results obtained on hexahedral meshes and the finite-element results obtained on tetrahedral meshes are qualitatively very similar. However, the finite-volume solutions obtained on tetrahedral meshes, shown in Fig. 10, are noticeably inferior to the others.

The tetrahedral meshes are generated directly from the hexahedral meshes by splitting each hexahedra into six tetrahedra with a consistent stencil. ${ }^{29}$ During this process, all the surface triangles have been generated by splitting the surface quadrilaterals in identical directions, thereby introducing a strong bias in the mesh that apparently has a stronger effect on the finite-volume solutions than on the finite-element solutions. Meshes with more random dissection of the hexahedra or meshes typically produced by unstructured grid generation, may mitigate the strong bias of these tetrahedral grids.

Diskin et al. ${ }^{28}$ reported odd-even decoupling for unweighted least-squares reconstruction with FUN3D for this case, which is indicated by the zigzag pattern in Fig. 10(a) contours. This odd-even decoupling can be addressed with a directional gradient along the structured grid lines or an approximate mapping 


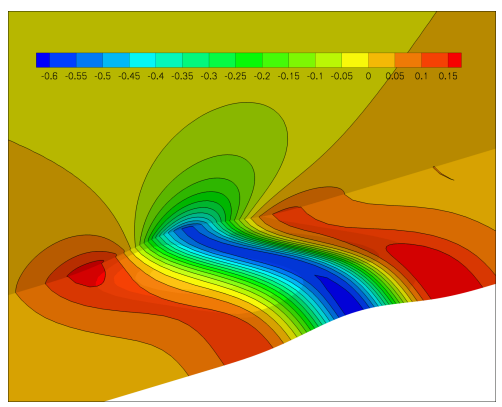

(a) 966 thousand node hexahedral mesh.

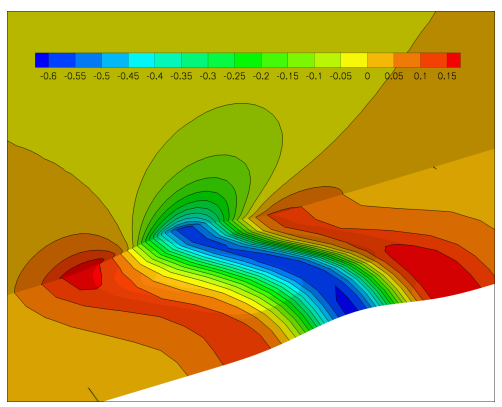

(b) 129 thousand hexahedral mesh.

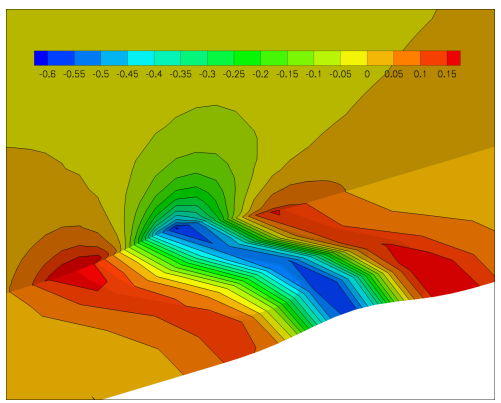

(c) 18 thousand hexahedral mesh.

Figure 8. Pressure coefficients from finite-volume scheme on hexahedral meshes.

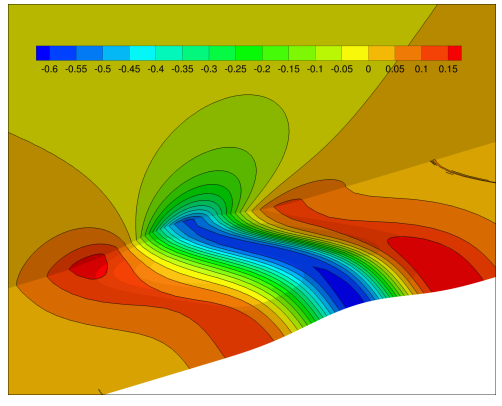

(a) 966 thousand tetrahedral mesh.

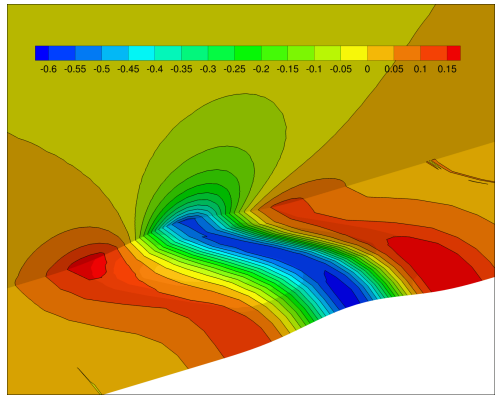

(b) 129 thousand tetrahedral mesh.

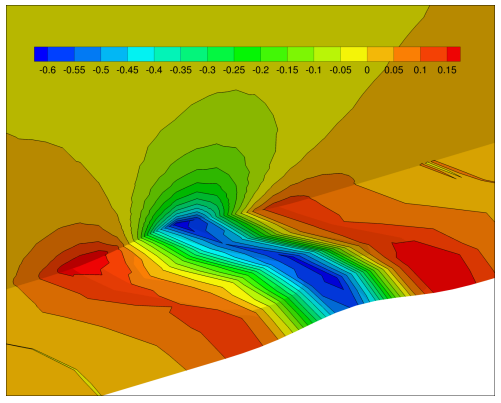

(c) 18 thousand tetrahedral mesh.

Figure 9. Pressure coefficients from finite-element scheme on tetrahedral meshes.

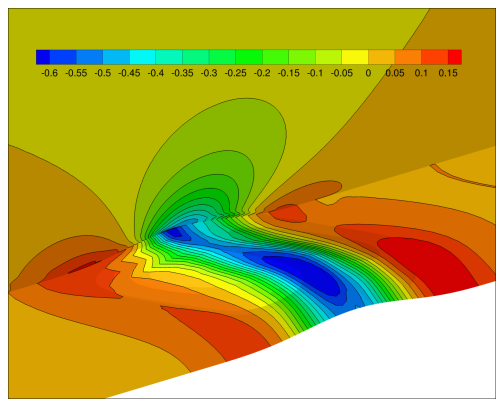

(a) 966 thousand tetrahedral mesh.

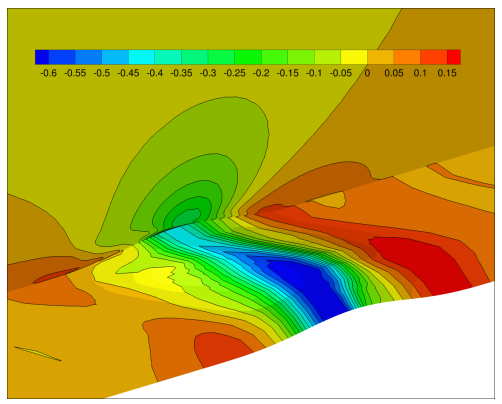

(b) 129 thousand tetrahedral mesh.

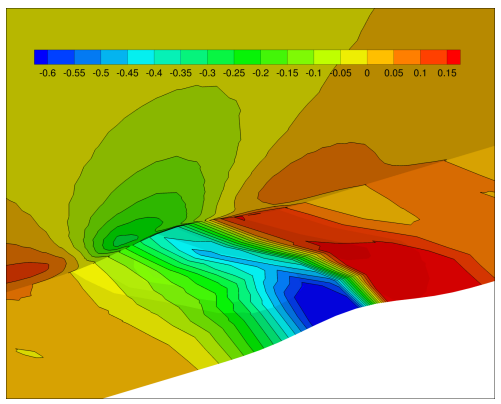

(c) 18 thousand tetrahedral mesh.

Figure 10. Pressure coefficients from finite-volume scheme on tetrahedral meshes.

technique. ${ }^{28}$ It should be noted that past experience and the other cases in this paper show that the finitevolume discretization on pure tetrahedral meshes typically yields good accuracy. The strongly biased grids with curvature in this case may be exacerbating the weaknesses of unweighted least-squares reconstruction. ${ }^{30}$

Surface pressure distributions along the $y=0.0$ symmetry plane are shown in Fig. 11. Figure 11(a) shows results obtained with the finite-volume discretization on hexahedral meshes ranging in size from 18 thousand nodes to 966 thousand nodes, as well as the finite-volume reference solution, which is computed on the mesh with almost 59 million nodes. Similar results are shown in Fig. 11(b) for finite-element solutions on the tetrahedral meshes. As seen in the figures, the finite-volume results on hexahedra and the finiteelement results on tetrahedra are virtually indistinguishable. In contrast, the finite-volume results obtained on tetrahedra in Fig. 11(c) show a substantive decline in accuracy compared to the others. The accuracy reduction appears to be a result of the odd-even decoupling seen in Fig. 10.

Velocity profiles, extracted along a vertical line extending vertically from $(1.2079,-0.1250,0.0)$ are shown in Fig. 12. Figure 12(a) shows results obtained on the 966 thousand hexahedral and tetrahedral meshes compared with the reference solution. As seen, the finite-element solution obtained on the tetrahedral 


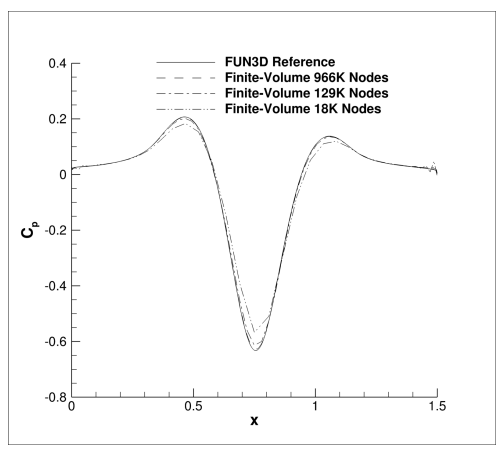

(a) Finite volume (hexahedra).

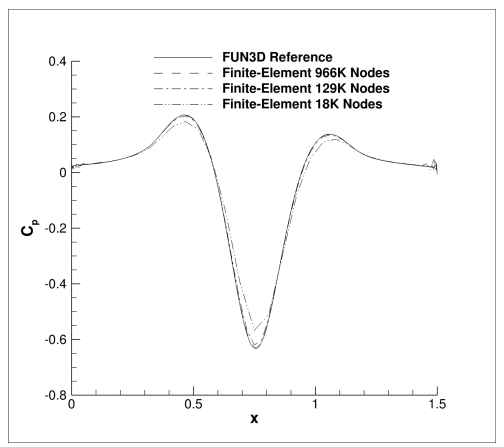

(b) Finite element (tetrahedra).

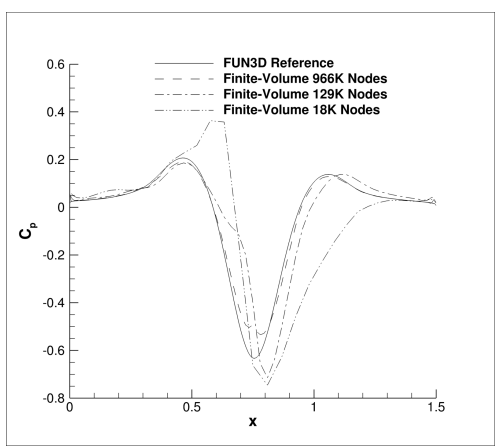

(c) Finite volume (tetrahedra).

Figure 11. Convergence of pressure coefficients along the $y=0$ symmetry plane.

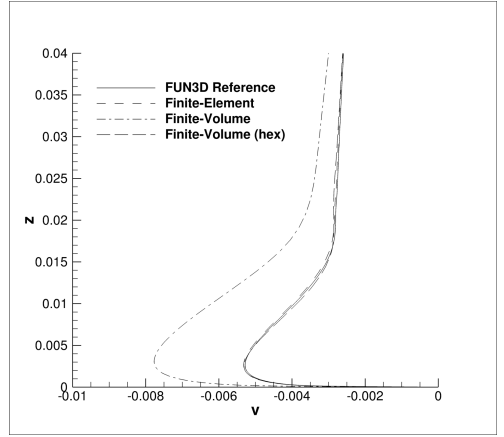

(a) 966 thousand mesh.

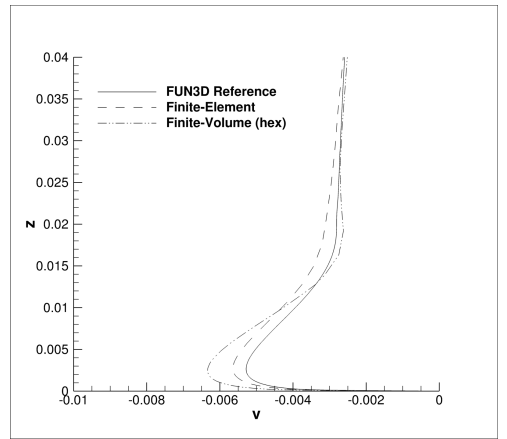

(b) 129 thousand mesh.

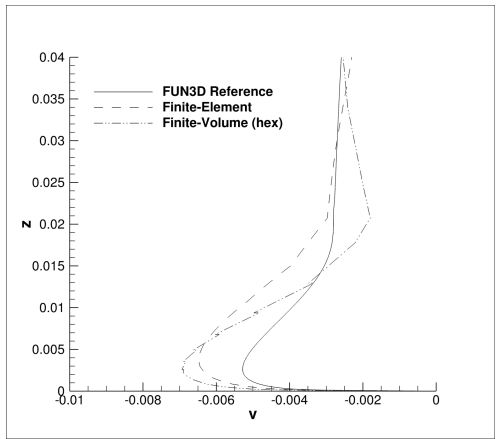

(c) 18 thousand mesh.

Figure 12. Profiles of $v$-velocity.

mesh and the finite-volume hexahedral mesh agree very well with the reference solution. The finite-volume solution on the tetrahedral mesh shows much more disparity. Profiles in Fig. 12(b) and Fig. 12(c) show similar results to the reference solutions with slight degradation as the mesh is coarsened. The finite-volume solutions obtained on tetrahedra have been omitted in Fig. 12(b) and (c) to provide a better view of the comparison between the finite-element scheme on tetrahedra and the finite-volume scheme on hexahedra. On the 129 thousand mesh, the finite-element scheme on tetrahedra appears to be somewhat closer to the reference solution near the wall than the finite-volume scheme on hexahedra, but this trend is reversed at the edge of the boundary layer. The odd-even decoupling seen in pressures of the finite-volume scheme on tetrahedral grids appear to also negatively impact the velocities.

Profiles of the turbulence working variable, along a vertical line emanating from $(1.2079,-0.1250,0.0)$, are shown in Fig. 13. As with the results presented for the velocity profiles, Fig. 13(a) demonstrates that the

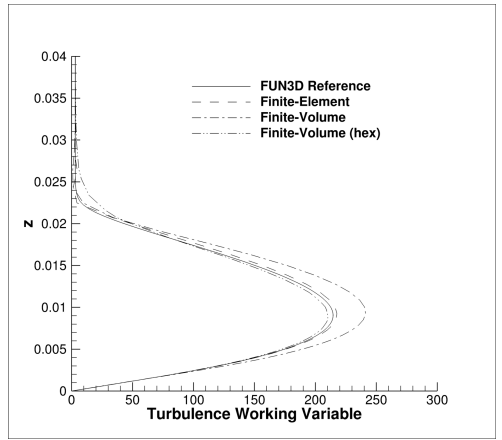

(a) 966 thousand mesh.

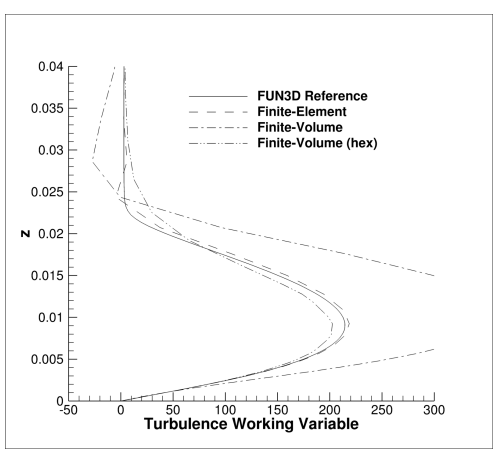

(b) 129 thousand mesh.

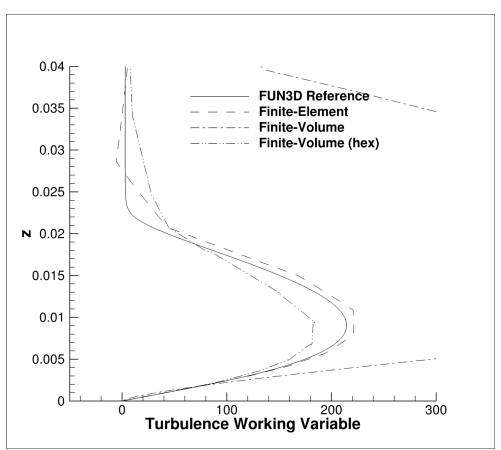

(c) 18 thousand mesh.

Figure 13. Profiles of turbulence working variable. 
results obtained using the finite-element discretization on the 966 thousand tetrahedral mesh agrees well with the reference solution, as does the finite-volume solution obtained on the 966 thousand hexahedral mesh. Again, the finite-volume solution obtained on the tetrahedral mesh is somewhat degraded when compared to the other solutions. Figure 13(b) shows similar results obtained on the 129 thousand meshes. In contrast to the velocity profiles, results from the finite-volume scheme on tetrahedra are partially shown in this figure, although they are clipped to facilitate better comparison between the other results. For this mesh density, the finite-element solution appears to be in slightly better agreement than the finite-volume scheme using hexahedra. On the coarsest mesh density, consisting of only 18 thousand nodes, the finite-element solution on tetrahedra continues to provide good results when compared to the much finer finite-volume reference solution on the hexahedral mesh. The finite-volume solution obtained on the coarsest tetrahedral mesh is clearly less accurate as the results are barely visible as they exceed the range in Fig. 13(b) and (c).

The final results for the swept-bump case depict the drag computed with each scheme on a sequence of continuously refined meshes to observe the effects of varying mesh density. Figure 14(a) shows the convergence of the total drag with mesh refinement. The finite-volume scheme on tetrahedra shows significantly more variation than the same scheme on hexahedra, or the finite-element scheme on tetrahedra. Examining the finite-volume results on tetrahedra compared with those for the hemisphere cylinder in Fig. 6, the variation in drag for this case is significantly higher than for the 3D hemisphere cylinder. The odd-even decoupling seen in pressures and velocities of the finite-volume scheme on tetrahedral grids appear to also impact the integrated drag convergence.

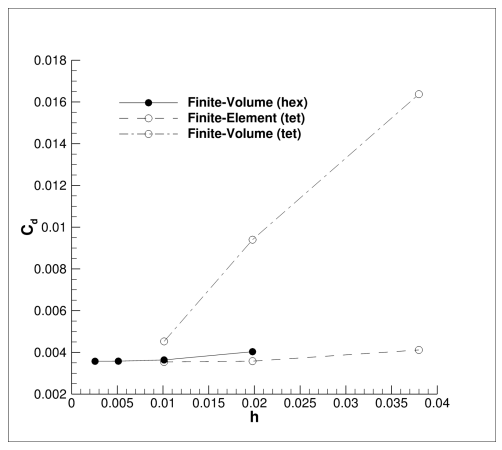

(a) Total drag.

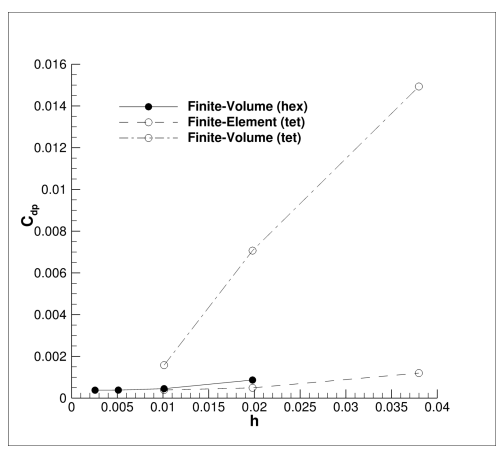

(b) Pressure drag.

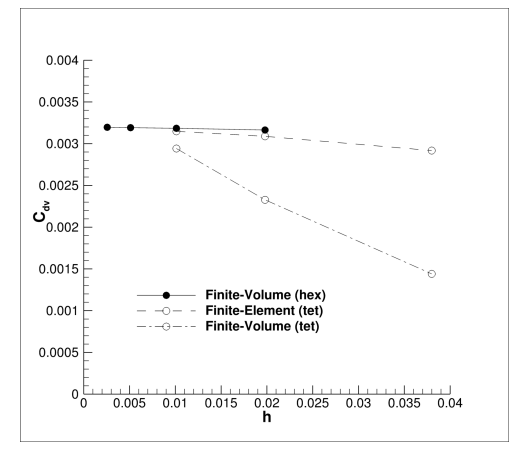

(c) Viscous drag.

Figure 14. Drag coefficients with mesh refinement.

Because of the wide variation in the drag results from the finite-volume scheme obtained on the tetrahedral meshes, Fig. 15 shows a view of the results from Figure 14, excluding the finite-volume solutions on the tetrahedral meshes with the largest variation. It appears that the solutions on the hexahedral meshes obtained with the finite-volume discretization and the finite-element solutions on tetrahedral meshes asymptote to similar values as the mesh is refined.

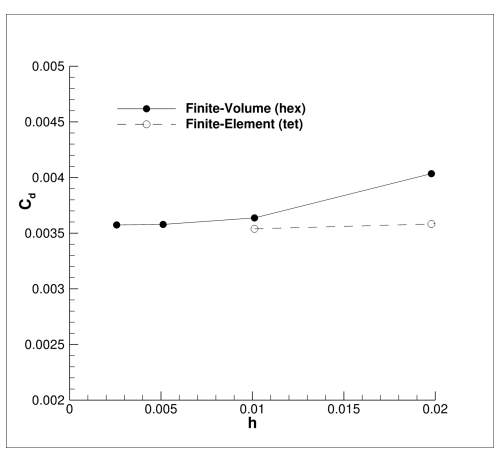

(a) Total drag.

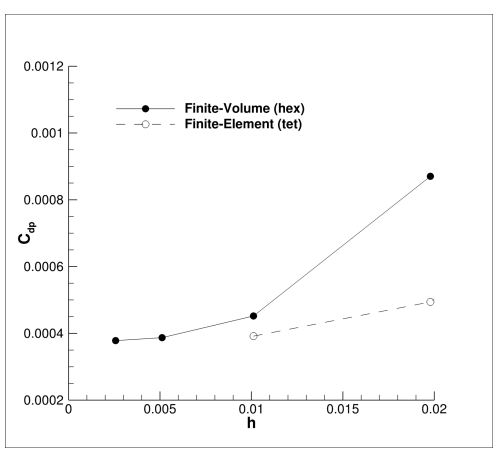

(b) Pressure drag.

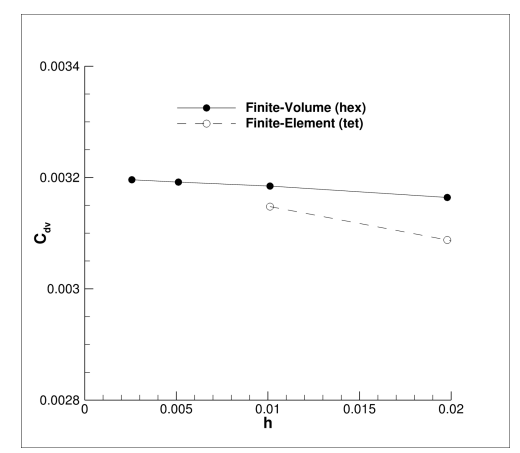

(c) Viscous drag.

Figure 15. Enhanced view of drag coefficients with mesh refinement. 


\section{III.C. 3D Supersonic Flow in a Square Duct}

Experimental data for this case is extracted from Davis and Gessner ${ }^{31}$ as provided by the TMR web site. This case models flow in a square duct that develops without strong shock waves. The lack of strong shocks focuses this example on the viscous development without overwhelming inviscid shock structures. The presence of turbulence-driven secondary flow is a stringent test for turbulence models to predict supersonic corner flows.

One quarter of the square domain cross section with internal duct width of $D$ is modeled by symmetry planes. A short symmetry boundary length of $1.26829 D$ is extended in front of a non-slip adiabatic wall of $52 \mathrm{D}$ in length. The inflow boundary is Riemann invariant to provide a uniform supersonic inflow of 3.9 Mach number and $520{ }^{\circ} \mathrm{R}$, which are also the reference conditions. The outflow boundary is extrapolation. The Reynolds number of 508,000 is based on $D$.

A Fortran code is provided by the TMR web site to generate a structured H-topology grid with clustering in the solid wall corner of the $0.5 D \times 0.5 D$ domain with coarsest spacing along the symmetry boundary condition intersection. Once the finest level0 grid is created, it is recursively coarsened by removing every other node in each coordinate direction, reducing cell counts by factors of eight. The quarter-domain structured grid provided is converted into an unstructured hexahedral grid format. Each hexahedral grid constructed from these structured grid is converted into tetrahedral grids with the method of Dompierre et al. ${ }^{29}$ Four grid levels with two element types are examined and their dimensions are given in Table 4. Each grid is either all hexahedra or all tetrahedra and the number of nodes is independent of element type. The coarsest hexahedral mesh is shown in Fig. 16.

Table 4. Committee supplied structured and hybrid grids.

\begin{tabular}{lrrr} 
Grid Level & Nodes & Hexahedra & Tetrahedra \\
\hline level3 & 53,125 & 48,384 & 290,304 \\
level2 & 405,769 & 387,072 & $2,322,432$ \\
level1 & $3,170,833$ & $3,096,576$ & $18,579,456$ \\
level0 & $25,068,577$ & $24,772,608$ & $148,635,648$
\end{tabular}

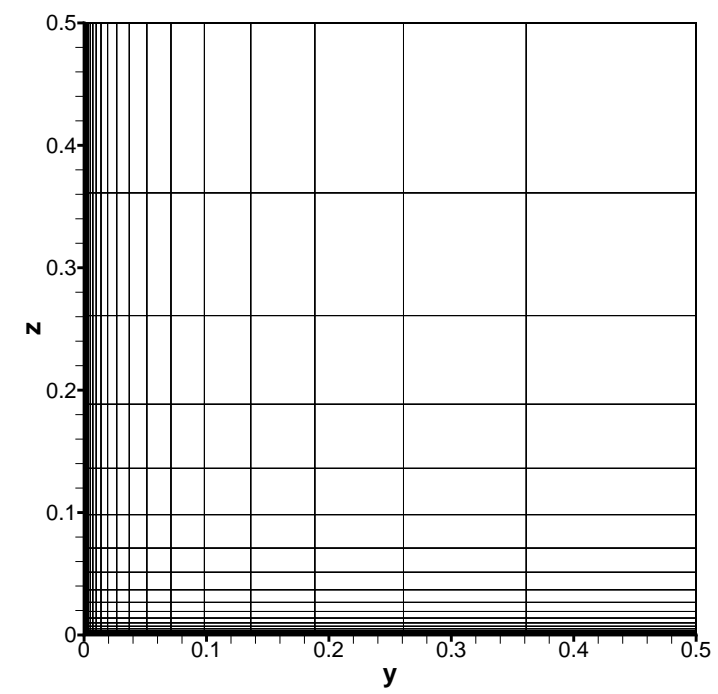

(a) $y-z$ view.

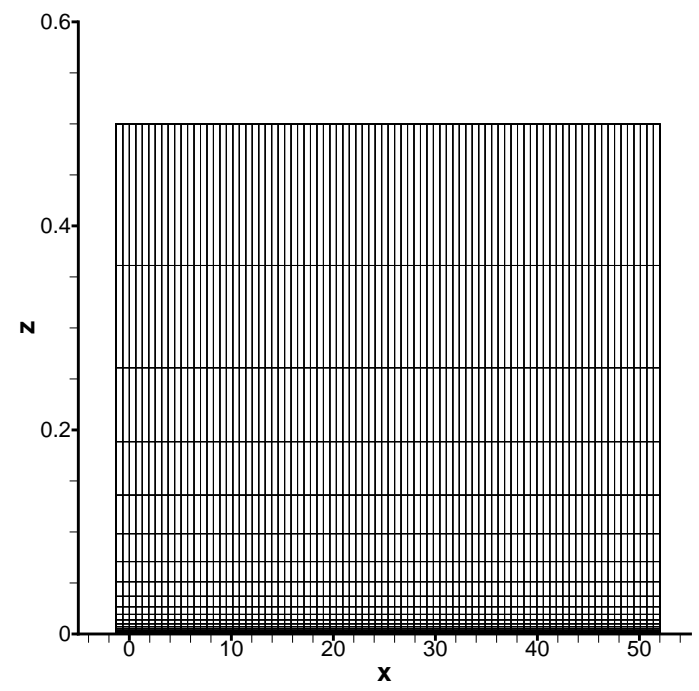

(b) $x-z$ view, $x$ scaled by $1 / 100$.

Figure 16. Supersonic square duct hexahedral level3 mesh.

First- and second-order convection (using reconstructed turbulence model working variable gradients) is utilized with the SA-neg and SA-neg-QCR2000. The RMS of all supersonic square duct residuals are reduced ten orders of magnitude. The hexahedral level0 SA-neg-QCR2000 with second-order turbulence working variable convection was restarted from the corresponding converged first-order convection case because initialization with reference conditions resulted in stalled convergence. All other cases were initialized 
with reference conditions.

The skin friction profile exhibited by SA-neg-QCR2000 in Fig. 17 and Fig. 18 is indicative of the secondary corner flow observed in the experiment. The SA-neg skin friction profile (Fig. 19) indicates a lack of secondary flow, which results in the poor prediction of experimental skin friction.

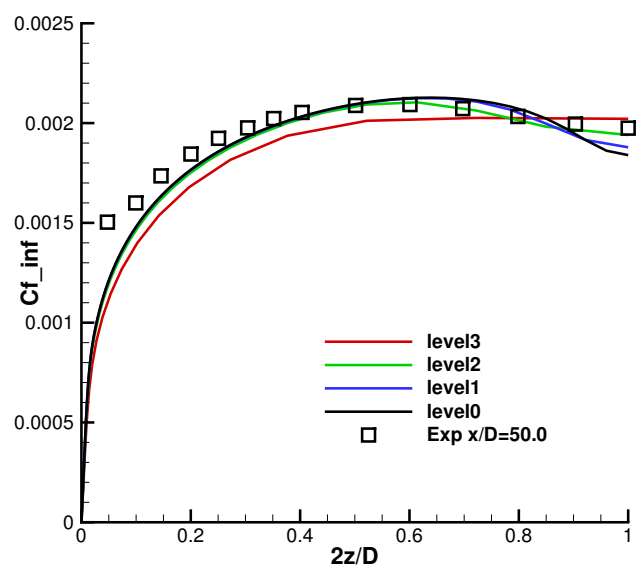

(a) First-order convection SA-neg-QCR2000.

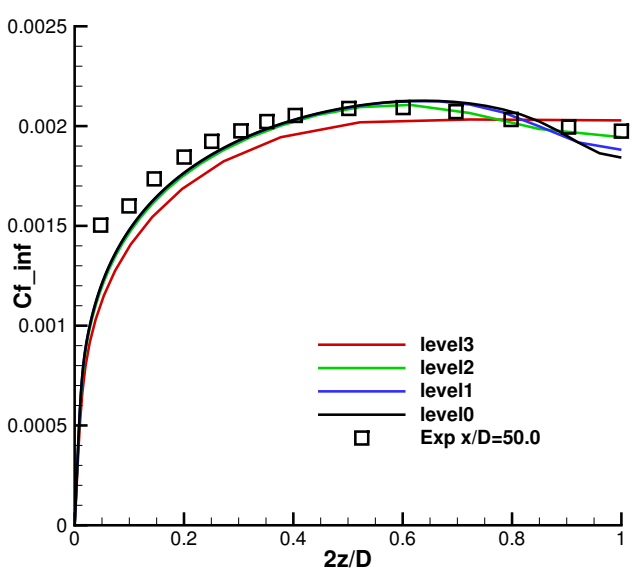

(b) Second-order convection SA-neg-QCR2000

Figure 17. Hexahedral mesh, supersonic square duct skin friction uniform grid refinement at $50 D$ station.

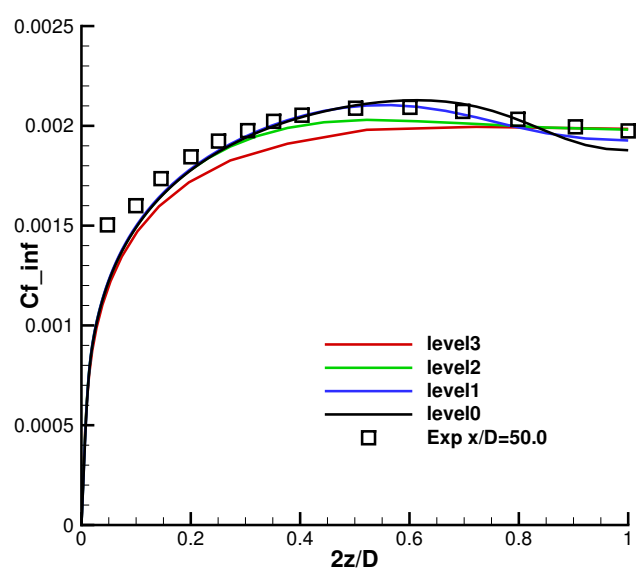

(a) First-order convection SA-neg-QCR2000.

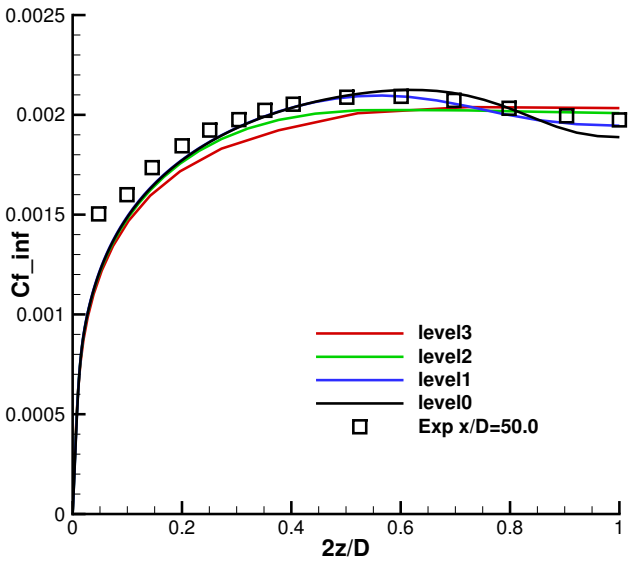

(b) Second-order convection SA-neg-QCR2000

Figure 18. Tetrahedral mesh, supersonic square duct skin friction uniform grid refinement at $50 D$ station.

SA-neg-QCR2000 (Fig. 17 and Fig. 18) appears to require a finer grid than SA-neg (Fig. 19) as inferred from the greater amount of variation between grids, particularly inflection in skin friction seen near the centerline of the finer two grids. Grid dependency trends are similar for the hexahedral (Fig. 17) and tetrahedral (Fig. 18) grids. First- and second-order convection show similar grid dependency trends. The difference between first- and second-order convection for a grid level is negligible. Differences can only be observed on the coarsest level3 tetrahedral grid.

SA-neg on hexahedral grids (Fig. 19) shows very little variation between grids, with the only difference shown by the centerline skin friction on the coarsest grid. This indicates that these grids may be sufficiently fine for this turbulence model. The SA-neg simulations appear to be grid resolved on this family of grids, but the SA-neg-QCR2000 simulations may require an increase in grid resolution to resolve the inflection in the skin friction profile near the duct centerline.

The drag coefficient on the square duct is shown in Fig. 20 as a function of characteristic $h$. Linear 


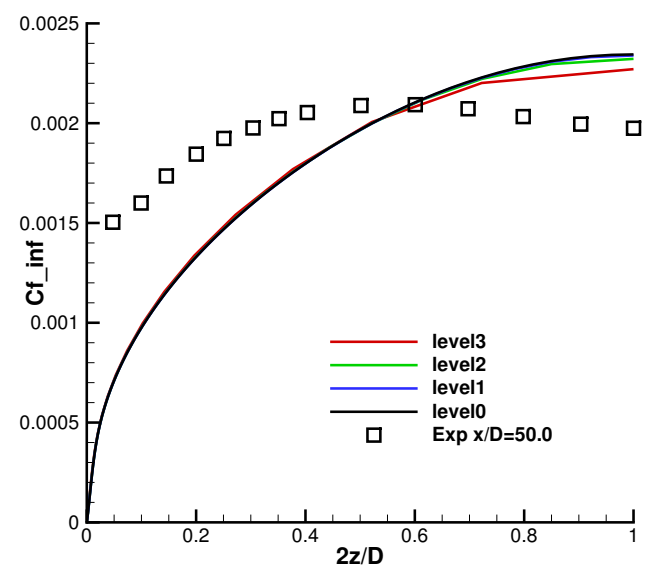

Figure 19. Hexahedral mesh, supersonic square duct skin friction uniform grid refinement at 50 $D$ station, first-order convection SA-neg.

trends indicate that a method presents first-order convergence in space. This characteristic $h$ is estimated with the number of nodes $N$ by $h=N^{-1 / 3}$. The two series of hex grids (square symbols) appear to be insensitive to the accuracy of the convection terms in the turbulence model for SA-neg-QCR2000. The two series of tetrahedral grids (triangle symbols) are more sensitive to the accuracy of the convection terms in the turbulence model. The second-order convection tetrahedral drag is near the hexahedral drag on equivalent grids. The first-order convection tetrahedral drag is lower than the hexahedral drag or secondorder convection tetrahedral drag, but appears to be approaching the same asymptotic value. The SA-neg drag is lower than that predicted by the SA-neg-QCR2000 model, which may be a result of the difference in secondary flow prediction indicated by the skin friction profiles.

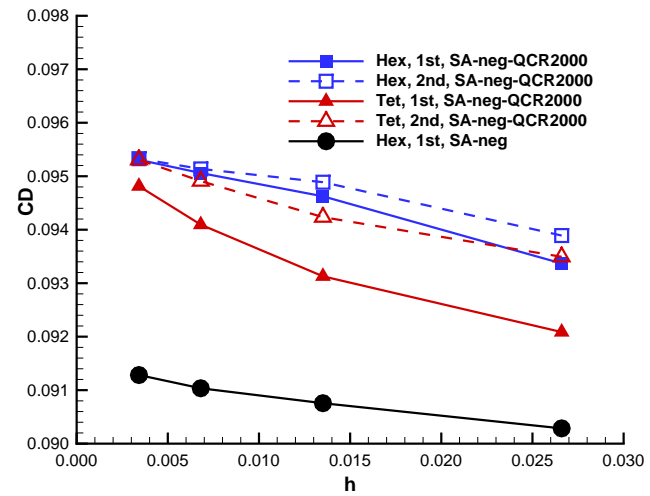

Figure 20. Supersonic square duct drag coefficient uniform grid refinement.

\section{Conclusions}

The hemisphere cylinder, bump, and square duct 3D cases from the TMR web site were presented. The finite-element formulation in FUN3D provided better comparisons to reference solutions than the finitevolume formulation. The 3D bump showed odd-even decoupling on tetrahedral grids created with a biased subdivision of hexahedral grids that polluted surface pressures and negatively impacted velocity and turbulence working variable profiles. Diskin et al. ${ }^{28}$ indicated this odd-even decoupling could be mitigated with different gradient reconstruction techniques for mean flow convection. The odd-even decoupling was not observed for the hemisphere cylinder or supersonic square duct tetrahedral grids. The supersonic duct showed 
very weak grid topology or turbulence convection order of accuracy dependence. SA-neg-QCR2000 resulted in improved prediction of experimental flow topology over SA-neg as indicated by skin friction comparisons to experiment. The secondary corner flows simulated by SA-neg-QCR2000 showed more grid dependence than SA-neg.

\section{Acknowledgments}

The authors would like to thank Jim Thomas, NASA Langley Distinguished Research Associate, Boris Diskin, National Institute of Aerospace, and Chris Rumsey, NASA Langley, for providing suggestions and feedback.

\section{References}

${ }^{1}$ Mavriplis, D. J., Vassberg, J. C., Tinoco, E. N., Mani, M., Brodersen, O. P., Eisfeld, B., Wahls, R. A., Morrison, J. H., Zickuhr, T., Levy, D., and Murayama, M., "Grid Quality and Resolution Issues from the Drag Prediction Workshop Series," AIAA Journal of Aircraft, Vol. 46, No. 3, May-June 2009, pp. 935-950.

${ }^{2}$ Levy, D. W., Laflin, K. R., Tinoco, E. N., Vassberg, J. C., Mani, M., Rider, B., Rumsey, C. L., Wahls, R. A., Morrison, J. H., Brodersen, O. P., Crippa, S., Mavriplis, D. J., and Murayama, M., "Summary of Data from the Fifth Computational Fluid Dynamics Drag Prediction Workshop," AIAA Journal of Aircraft, Vol. 51, No. 4, July-Aug. 2014, pp. $1194-1213$.

${ }^{3}$ Morrison, J. H., "Statistical Analysis of the Fifth Drag Prediction Workshop Computational Fluid Dynamics Solutions," AIAA Journal of Aircraft, Vol. 51, No. 4, July-Aug. 2014, pp. 1214-1222.

${ }^{4}$ Diskin, B., Thomas, J. L., Rumsey, C. L., and Schwöppe, A., "Grid Convergence for Turbulent Flows," AIAA Paper 2015-1746, 2015.

${ }^{5}$ http://turbmodels.larc.nasa.gov [cited 1 November 2015].

${ }^{6}$ Rumsey, C. L., Smith, B. R., and Huang, G. P., "Description of a Website Resource for Turbulence Modeling Verification and Validation," AIAA Paper 2010-4742, 2010.

${ }^{7}$ Rumsey, C. L., "Turbulence Modeling Verification and Validation," AIAA Paper 2014-201, 2014.

${ }^{8}$ Anderson, W. K. and Bonhaus, D. L., "An Implicit Upwind Algorithm for Computing Turbulent Flows on Unstructured Grids," Computers and Fluids, Vol. 23, No. 1, 1994, pp. 1-22.

${ }^{9}$ Nielsen, E. J., Aerodynamic Design Sensitivities on an Unstructured Mesh Using the Navier-Stokes Equations and a Discrete Adjoint Formulation, Ph.D. thesis, Virginia Polytechnic Institute and State University, 1998.

${ }^{10}$ Roe, P. L., "Approximate Riemann Solvers, Parameter Vectors, and Difference Schemes," Journal of Computational Physics, Vol. 43, No. 2, Oct. 1981, pp. 357-372.

${ }^{11}$ Burg, C. O. E., Sheng, C., Newman, III, J. C., Brewer, W., Blades, E., and Marcum, D. L., "Verification and Validation of Forces Generated by an Unstructured Flow Solver," AIAA Paper 2003-3983, 2003.

${ }^{12}$ Burg, C. O. E., "Higher Order Variable Extrapolation For Unstructured Finite Volume RANS Flow Solvers," AIAA Paper 2005-4999, 2005.

${ }^{13}$ Diskin, B., Thomas, J. L., Nielsen, E. J., Nishikawa, H., and White, J. A., "Comparison of Node-Centered and CellCentered Unstructured Finite-Volume Discretizations: Viscous Fluxes," AIAA Journal, Vol. 48, No. 7, July 2010, pp. 1326-1338.

${ }^{14}$ Spalart, P. R. and Allmaras, S. R., "A One-Equation Turbulence Model for Aerodynamic Flows," La Recherche Aérospatiale, Vol. 1, 1994, pp. 5-21.

${ }^{15}$ Allmaras, S. R., Johnson, F. T., and Spalart, P. R., "Modifications and Clarifications for the Implementation of the Spalart-Allmaras Turbulence Model," Seventh International Conference on Computational Fluid Dynamics (ICCFD7), 2012.

${ }^{16}$ Spalart, P. R., "Strategies for Turbulence Modelling and Simulations," International Journal of Heat and Fluid Flow, Vol. 21, No. 3, June 2000, pp. 252-263.

${ }^{17}$ Eisenstat, S. C., Elman, H. C., and Schultz, M. H., "Variational Iterative Methods for Nonsymmetric Systems of Linear Equations," SIAM Journal on Numerical Analysis, Vol. 2, April 1983, pp. 345-357.

${ }^{18}$ Pandya, M. J., Diskin, B., Thomas, J. L., and Frink, N. T., "Improved Convergence and Robustness of USM3D Solutions on Mixed Element Grids," AIAA Paper 2015-1747, 2013.

${ }^{19}$ Brooks, A. N. and Hughes, T. J. R., "Streamline Upwind/Petrov-Galerkin Formulation for Convection Dominated Flows with Particular Emphasis on Incompressible Navier-Stokes Equations," Computer Methods in Applied Mechanics and Engineering, Vol. 32, No. 1-3, Sept. 1982, pp. 199-259.

${ }^{20}$ Shakib, F., Hughes, T. J. R., and Johan, Z., "A New Finite-Element Formulation for Computational Fluid Dynamics: X. The Compressible Euler and Navier-Stokes Equations," Computer Methods in Applied Mechanics and Engineering, Vol. 89, No. 1-3, Aug. 1991, pp. 141-219.

${ }^{21}$ Hughes, T. J. R., Franca, L. P., and Hulbert, G. M., "A New Finite-Element Formulation for Computational Fluid Dynamics: VIII. The Galerkin Least Squares Method for Advective-Diffusion Equations," Computer Methods in Applied Mechanics and Engineering, Vol. 73, No. 2, May 1989, pp. 173-189.

${ }^{22}$ Bazilevs, Y. and Akkerman, I., "Large Eddy Simulation of Turbulent Taylor-Couette Flow Using Isogeometric Analysis and the Residual-Based Variational Multiscale Method," Journal of Computational Physics, Vol. 229, No. 9, May 2010, pp. 3402-3414.

${ }^{23}$ Burgess, N. and Glasby, R., "Advances in Numerical Methods for CREATE-AV Analysis Tools," AIAA Paper 2014-417, 2014. 
${ }^{24}$ Saad, Y. and Schultz, M. H., "GMRES: A Generalized Minimum Residual Algorithm for Solving Nonsymmetric Linear Systems," SIAM Journal of Scientific and Statistical Computing, Vol. 7, 1986, pp. 856-869.

${ }^{25}$ Saad, Y., Iterative Methods for Sparse Linear Systems, Society for Industrial and Applied Mathematics, Philadelphia, PA, USA, 2nd ed., 2003.

${ }^{26}$ Anderson, W. K., Ahrabi, B. R., and Newman III, J. C., "Finite-Element Solutions for Turbulent Flow over the NACA 0012 Airfoil," AIAA Paper 2015-1531, 2015.

${ }^{27}$ Hsieh, T., "An Investigation of Separated Flow about a Hemisphere-Cylinder at 0- to 19-deg Incidence in the Mach Number Range from 0.6 to 1.5," Arnold Engineerig Development Center, Arnold Air Force Base AEDC-TR-76-112, Nov. 1976.

${ }^{28}$ Diskin, B., Thomas, J. L., Pandya, M. J., and Rumsey, C. L., "Reference Solutions for Benchmark Turbulent Flows in Three Dimensions," AIAA SciTech Forum and Exposition (SciTech 2016), American Institute of Aeronautics and Astronautics, Reston, VA (submitted for publication).

${ }^{29}$ Dompierre, J., Labbé, P., Vallet, M.-G., and Camarero, R., "How to Subdivide Pyramids, Prisms, and Hexahedra into Tetrahedra," 8th International Meshing Roundtable, 1999.

${ }^{30}$ Diskin, B. and Thomas, J. L., "Comparison of Node-Centered and Cell-Centered Unstructured Finite-Volume Discretizations: Inviscid Fluxes," AIAA Journal, Vol. 49, No. 4, April 2011, pp. 1326-1338.

${ }^{31}$ Davis, D. O. and Gessner, F. B., "Further Experiments on Supersonic Turbulent Flow Development in a Square Duct," AIAA Journal, Vol. 27, No. 8, Aug. 1989, pp. 1023-1030. 